\title{
ON LINEAR PLANES
}

\author{
AVINASH SATHAYE
}

\begin{abstract}
ABSTRACr. A linear plane over a ground field $k$ is an algebraic surface in affine 3-space over $k$ which is biregular to the affine plane and whose equation is linear in one of the three variables of the 3-space. In this note we give a concrete description of a linear plane over a field of characteristic zero, thereby proving it to be an embedded plane, i.e. we show that by an automorphism of the affine 3-space, it can be transformed to a coordinate plane.
\end{abstract}

1. Introduction. Let $A(n, k)$ denote a polynomial ring in $n$-variables over a domain $k$, which we (geometrically) call the affine $n$-space over the ground domain $k$. By a hypersurface in $A(n, k)$ we mean any nonunit principal ideal, say $(f), f \in A(n, k)$. If there is no confusion we will simply say that " $f$ is a hypersurface in $A(n, k)$ ".

Now let $k$ be a field. A hypersurface $f$ is defined to be

(1) a hyperplane over $k$ in $A(n, k)$, if $A(n, k) /(f) \approx A(n-1, k)$;

(2) a general hyperplane over $k$, if $A(n, \bar{k}) /(f+\lambda) \approx A(n-1, \bar{k})$ for all $\lambda \in \bar{k}$, where $\bar{k}=$ the algebraic closure of $k$;

(3) a generic hyperplane over $k$, if $A(n, k(T)) /(f-T) \approx A(n-1, k(T))$, where $T$ is an indeterminate over $k$;

(4) an embedded hyperplane over $k$, if

$$
A(n, k)=B[f], \quad \text { where } B \approx A(n-1, k) ;
$$

(5) a linear hypersurface over $k$, if $A(n, k)=B[Z]$, with $B \approx A(n-1, k)$ and $Z \in A$ such that $f=a Z+b$ with $a, b \in B, a \neq 0$;

(6) a linear hyperplane over $k$, if $f$ is both a linear hypersurface and a hyperplane.

As usual, when $n \leqslant 3$ we drop "hyper" and for $n=2$ replace the words surface and plane by curve and line respectively.

Now assume that either

(*) $f$ is a hyperplane, or (as possibly stronger hypothesis)

$(* *) f$ is a linear hyperplane.

For each $n \geqslant 1$, the following questions arise naturally.

$\mathrm{Q}(1 . n)$. Is $f$ a general hyperplane over $k$ ?

$\mathrm{Q}(2 . n)$. Is $f$ a generic hyperplane over $k$ ?

$\mathrm{Q}(3 . n)$ (Epimorphism problem). Is $f$ an embedded hyperplane over $k$ ?

Received by the editors May 19, 1975.

AMS (MOS) subject classifications (1970). Primary 13B15, 14E25; Secondary 13F20, 14E35.

Key words and phrases. Biregular hyperplane, generic hyperplane, embedded hyperplane, epimorphisms of polynomial rings, automorphisms of polynomial rings. 
Note that "yes" to $\mathrm{Q}(3 . n)$ clearly implies "yes" to $\mathrm{Q}(1 . n)$ and $\mathrm{Q}(2 . n)$. Also note that for $n=1$, the answer is (trivially) "yes" to all questions.

For $n=2$ and char $k=0$, Abhyankar and Moh gave an affirmative answer to all questions by the Epimorphism Theorem, which is an affirmative answer to $\mathrm{Q}(3,2)$, with hypothesis $(*)$ [AM].

For $n=2$ with hypothesis (*), and char $k=p \neq 0, \mathrm{Q}(3.2)$ and $\mathrm{Q}(2.2)$ are known to have a common counterexample, namely

$$
f=y^{p^{2}}-x-x^{2 p} \in k[x, y] \approx A(2, k) .
$$

It is easy to see that the same example serves as a counterexample to $Q(2 . n)$ and $\mathrm{Q}(3 . n)$ in general. We point out that as yet no counterexamples to $\mathrm{Q}(1.2)$ seem to be known.

With hypothesis $(* *)$ and $n=2$, however, it is trivial to show that the answer to all questions is affirmative even in characteristic $p \neq 0$.

In this note, we prove the first step for $n=3$, by proving the

THEOREM. If char $k=0$, then any linear plane in $A(3, k)$ is an embedded plane.

I would like to thank Professor Heinzer and Mr. Gurjar for stimulating conversations on this problem.

In [R] Peter Russell has established our Lemma 3 in any characteristic, thereby establishing the Theorem for arbitrary field $k$.

2. Proof of the Theorem. First we introduce some notation to be fixed throughout.

Notation. Write $A(3, k)=C[Z]$ such that $H \in C[Z]$ is a linear plane linear in $Z$ and $C \approx A(2, k)$. If $K$ denotes the quotient field of $C$ then $K[Z]=K[H]$. Thus there is a retract $\psi: K[Z] \rightarrow K$ with kernel $H$. Write

$$
H=g Z-f ; \quad g, f \in C \text {. }
$$

Then $\psi(Z)=f / g$. Restriction of $\psi$ to $C[Z]$ gives a map $C[Z] \rightarrow C[f / g]$ which is the identity map on $C$. We denote $C[f / g] \approx C[Z] /(H)$ by $B$. By hypothesis $B \approx A(2, k) \approx C$.

The theorem will be proved, when we show $H$ to be an embedded plane over $k$.

LEMMA 1. Assume that $k$ is of arbitrary characteristic $p$. If $f_{1}, f_{2}$ are two lines in $C$ such that $\left(f_{1}, f_{2}\right) C=C$ then there exist $c, d \in k$ such that

$$
f_{2}=c f_{1}+d, \quad c \neq 0 .
$$

Proof. Note that the only units modulo a line are constants (nonzero elements in the image of $k$ modulo the line). Since $f_{2}$ is a unit modulo $f_{1}$, we get

$$
f_{2}=c_{1} f_{1}+d_{1}, \quad 0 \neq c_{1} \in C, d_{1} \in k
$$

Similarly we can write

$$
f_{1}=c_{2} f_{2}+d_{2}, \quad 0 \neq c_{2} \in C, d_{2} \in k
$$

Comparing degrees with respect to any choice of $x, y$ with $C=k[x, y]$, we easily see from the above two equations that

$$
\text { degree } f_{1}=\text { degree } f_{2} \text { and degree } c_{1}=\text { degree } c_{2}=0 \text {. }
$$

The desired equation now follows. 
Corollary 1. Let $h \in k[x, y]=C$ and $k^{\prime}$ a separable extension of $k$. Assume that

$$
h=\prod_{1}^{s} u_{i}^{r_{i}}, \quad r_{i}>0,
$$

such that $u_{i} \in k^{\prime}[x, y] \approx A\left(2, k^{\prime}\right)$ are distinct lines over $k^{\prime}$. Moreover assume that $\left(u_{i}, u_{j}\right) k^{\prime}[x, y]=k^{\prime}[x, y]$, for $i \neq j$. Then there exist $c_{i}, d_{i} \in k^{\prime}$ and $u \in k[x, y]$ such that

$$
u_{i}=c_{i}\left(u+d_{i}\right)
$$

Further, $\Pi_{1}^{s} c_{i}^{r_{i}}=c$ is in $k$ and hence

$$
h=c \prod_{1}^{s}\left(u+d_{i}\right)^{r_{i}} .
$$

Proof. From Lemma 1 we can certainly find $u \in k^{\prime}[x, y]$ such that $(*)$ is satisfied. We start with such a choice and modify it to get it to be in $k[x, y]$.

By making an automorphism of $k[x, y]$ we can assume that the coefficient of the top $y$-degree in $u$ is some nonzero $e \in k^{\prime}$; and replacing $u$ by $u / e$ we get that $u$ is monic in $y$.

We will now show that all coefficients of $u$ except possibly the constant term belong to $k$.

Let $\sigma$ be an isomorphism of $k^{\prime} / k$. By $\sigma(u)$ we shall denote the result of acting $\sigma$ on all coefficients of $u$.

If $u \in k[x, y]$, then we are finished with the proof. Otherwise we can choose an isomorphism of $k^{\prime} / k$ such that $\sigma(u) \neq u$. By the expression of $h$, we get $\sigma(u)=a u+b, a, b \in k^{\prime}$.

Since $\sigma(u), u$ are both monic in $y$, we get $a=1$. Thus $\sigma(u)-u \in k^{\prime}$.

Since this is true for any $k$-isomorphism of $k^{\prime}$ and since $k^{\prime} / k$ is separable, we get that all coefficients of $u$ but the constant term $d$ say, belong to $k$.

Replacing $u$ by $u-d$, we get the desired expression because $c \in k$ is obvious by comparing the top degree coefficient of $y$ on both sides.

LEMMA 2. Let $k^{\prime} / k$ be a separable algebraic extension. If $u \in k[x, y]=C$ $\subset k^{\prime}[x, y]$ is a line over $k^{\prime}$, then $u$ is already a line over $k$.

Proof. Let $R=k[x, y] /(u)$ and $R^{\prime}=k^{\prime}[x, y] /(u)$. As usual, we may assume $k \subset R \subset R^{\prime}$ and $k^{\prime} \subset R^{\prime}$ by identifying isomorphic rings. Further, by extending $k^{\prime}$ if necessary we may assume $k^{\prime}$ is Galois over $k$.

Let $a \in R$ be algebraic over $k$. By assumption $R^{\prime}=k^{\prime}[t]$ for some $t$. Hence $a \in k^{\prime}$. Choose $h \in k[x, y]$ to be some preimage of $a$. If $a \neq 0$, then $h$ is unit modulo $(u) k^{\prime}[x, y]$ and hence a constant modulo $(u) k^{\prime}[x, y]$, i.e.

$$
h=u(x, y) p(x, y)+a^{*}, \quad a^{*} \in k^{\prime}, p(x, y) \in k^{\prime}[x, y] .
$$

Let $\sigma$ be any $k$-automorphism of $k^{\prime}$. Then applying $\sigma$ to (1)

$$
h=u(x, y) \sigma(p(x, y))+\sigma\left(a^{*}\right) .
$$

Subtracting (1) from (2) we conclude that $u(x, y)$ divides $a^{*}-\sigma\left(a^{*}\right)$ in $k^{\prime}[x, y]$. Thus $a^{*}=\sigma\left(a^{*}\right)$. Since this holds for each $\sigma, a^{*}$ is fixed by each member of the Galois group and hence $a^{*} \in k$.

Thus $k$ is relatively aigebraically closed in $R$. It is easy to see that we can 
replace $k^{\prime}$ by some finite extension $k^{*}$ of $k$ with $k^{*} \subset k^{\prime}$ such that $u \in$ $k^{*}[x, y]$ is a line over $k^{*}$, since we need to include in $k^{*}$ only the coefficients of polynomials expressing images of $x, y$ modulo $u$ in terms of $t$ and $t$ in terms of images of $x, y$ modulo $u$. Since $R$ is clearly normal (say by the Jacobian criterion) we can apply (2.9) of [AEH] to get that $R=k\left[t^{*}\right]$ for some $t^{*} \in R$. Thus $u \in k[x, y]$ is a line over $k$.

REMARK. If $K$ is a field of characteristic zero and $u \in K[x, y] \approx A(2, K)$ is a line over $K$, then there exists a $v \in K[x, y]$ such that $v$ is in the ring generated by the coefficients of $u$ and $K[u, v]=K[x, y]$.

This version of the Epimorphism Theorem can be deduced from [AM] by observing that $v$, in their terminology, is an "approximate root" of $u$ and hence has the above stated property.

Thus in the above Lemma 2 , we can find $v \in k[x, y]$ such that $k^{\prime}[u, v]=k^{\prime}[x, y]$. Writing $x, y$ as polynomials in $u, v$ over $k^{\prime}$ and taking "trace" it is easy to check that then $k[x, y]=k[u, v]$.

LEMMA 3. With the basic notations introduced at the beginning of this section we get the following. If char $k=0$, then there is an embedded line $u$ in $C$ over $k$ such that

$$
g=c \prod_{1}^{s}\left(u+d_{i}\right)^{r_{i},}, \quad r_{i}>0,
$$

where $c \in k$ and $\left\{d_{i}\right\}$ are distinct elements algebraic over $k$.

PROof. First suppose that the result is already true when $k$ is replaced by its algebraic closure, say $k^{\prime}$. In view of Corollary $1, u$ may be assumed to be in $k[x, y]$ and in view of Lemma $2, u$ is an embedded line over $k$. Thus we may assume that $k$ is algebraically closed.

Let $\mathfrak{N}=\left\{M_{1}, \ldots, M_{s}\right\}$ be the set of all maximal ideals in $C$ containing $f$, g. Clearly $B / M_{i} B \approx\left(C / M_{i}\right)[Z] \approx k[Z]$. Hence by the Epimorphism Theorem [AM] each $M_{i} B$ is an embedded line over $k$ (in $B$ ). Let $u_{i} B=M_{i} B$. Clearly $g$ has a factorization in $B$,

$$
g=\prod_{1}^{s} u_{i}^{r_{i}}, \quad r_{i}>0 .
$$

Also, it is clear from $u_{i} B=M_{i} B$ that $\left(u_{i}, u_{j}\right) B=B$, if $i \neq j$.

The expression for $g$ follows by applying Corollary 1 (with $k=k^{\prime}$ ). It remains to check that $u$ is already a line over $k$ in $C$.

Choose $\lambda \in k$ such that $u+\lambda \neq u+d_{i}$ for $i=1, \ldots, s$. We see that the image of $f / g$ modulo $(u+\lambda) B$ is contained in the image of $C$ modulo $C \cap(u+\lambda) B$, and hence $B /(u+\lambda) B=C /(u+\lambda) C$.

Now $u+\lambda$ is clearly an embedded line over $k$ in $B \approx A(2, k)$, and hence we get

$$
B /(u+\lambda) B \approx A(1, k) \approx C /(u+\lambda) C .
$$

Then $u+\lambda$ is an embedded line in $C$ over $k$ and hence so is $u$.

Lemma 4. Let $g$ be as in the conclusion of Lemma 3. Let $v \in C$ such that $k[u, v]=C$. Let $f=a_{0}+a_{1} v+\cdots+a_{n} v^{n}, a_{i} \in k[u]$. Then:

(1) $a_{1}$ is a unit in $k[u]$ modulo $g$. 
(2) $a_{2}, \ldots, a_{n}$ are nilpotent in $k[u]$ modulo $g$.

Proof. Let $k^{\prime}$ be the algebraic closure of $k$. Clearly, we only need to show that

$$
\begin{gathered}
a_{1} \notin\left(u+d_{i}\right) k^{\prime}[u], \text { for } i=1, \ldots, s ; \text { and } \\
a_{j} \in\left(u+d_{i}\right) k^{\prime}[u], \text { for } i=1, \ldots, s \text { and } j=2, \ldots, n .
\end{gathered}
$$

Since the hypothesis about $f, g, u$ and $H$ is unchanged if $k$ is replaced by $k^{\prime}$ we may assume that $k=k^{\prime}$, i.e. $k$ is algebraically closed.

Let, as in Lemma $3, M_{i}=\left(u+d_{i}\right) B \cap C$ and let $M_{i}^{*} \in C[Z]$ be the preimages of $M_{i}$ under $\psi$. Then it is clear that

$$
M_{i}^{*}=\left(u+d_{i}, g Z-f\right) C[Z]=\left(u+d_{i}, f\right) C[Z] .
$$

Hence we get that

$$
M_{i}=\left(u+d_{i}, f\right) C=M_{i}^{*} \cap C .
$$

Thus $f$ generates a maximal ideal in $C$ modulo $\left(u+d_{i}\right)$ and hence the image of $f$ in the canonical homomorphism $C \rightarrow C /\left(u+d_{i}\right)$ is a ring generator of $C /\left(u+d_{i}\right)$ over $k$. Since image of $v$ has the same property $\left(1^{*}\right)$ and $\left(2^{*}\right)$ are easily seen.

REMARK. In the above proof, if we simply assume $u \in C$ to be a line over $k$ (not necessarily embedded, as may happen in characteristic $p \neq 0$ ) then we still get that the image of $f$ modulo $\left(u+d_{i}\right)$ in $C$ is a ring generator.

Moreover, if $Q(1,2)$ could be answered in the affirmative in characteristic $p \neq 0$, then one could modify Lemma 3 to prove that $u+d_{i}$ is a line in $k[x, y]$ over $k$ (if $k$ is algebraically closed).

Now let $S$ be any ring and $S[V]$ a polynomial ring over $S$. A sequence $\left(c_{0}\right.$, $\left.c_{1}, \ldots, c_{n}\right)$ in $S$ is said to be a generator sequence if

(1) $c_{1}$ is a unit in $S$, and

(2) $c_{2}, \ldots, c_{n}$ are nilpotent in $S$.

Lemma 5. Let $S[V]$ be as above and let $h=c_{0}+c_{1} V+\cdots+c_{n} V^{n} \in$ $S[V]$ where $\left(c_{0}, c_{1}, \ldots, c_{n}\right)$ is a generator sequence. Then there exists a generator sequence $\left(0, c_{1}^{*}, \ldots, c_{n}^{*}\right)$ in $S$ and some $c \in S$ such that

$$
h=c_{1}^{*}(V+c)+\cdots+c_{n}^{*}(V+c)^{n} .
$$

In other words,

$$
h=(V+c)(\text { unit in } S[V])
$$

and hence

$$
(V+c)=h(\text { unit in } S[V]) \text {. }
$$

REMARK. The reason to call $\left(c_{0}, c_{1}, \ldots, c_{n}\right)$ a generator sequence is that the corresponding $h=c_{0}+c_{1} V+\cdots+c_{n} V^{n}$ is a ring generator of $S[V]$ over $S$.

If $h$ is a ring generator then it is easy to check that $\left(c_{0}, c_{1}, \ldots, c_{n}\right)$ is a generator sequence. To see the converse, let $T$ be an indeterminate over $S[V]$. Apply the Lemma to $h-T$ and $S[T]$ in place of $h$ and $S$, to get some $c \in S[T]$ such that $h-T \in(V+c) S[T][V]$.

Let $\sigma: S[V][T] \rightarrow S[T]$ be defined by $\sigma(V)=-c, \sigma(T)=T$ and $\sigma(s)=s$ 
for $s \in S$. The restriction of $\sigma$ to $S[V]$ is an $S$-isomorphism taking $h$ to $T$. It follows that $S[V]=S[h]$.

PROOF. For any generator sequence $\left(e_{0}, \ldots, e_{n}\right)$ in $S$ we define a new generator sequence $\left(\tau\left(e_{0}\right), \ldots, \tau\left(e_{n}\right)\right)$ as follows.

Let $e=e_{0} e_{1}^{-1}$. Write $\tau(V)=V+e$. Then $h(V)$ has a unique expression

$$
h(V)=e_{0}^{\prime}+e_{1}^{\prime}(V+e)+\cdots+e_{n}^{\prime}(V+e)^{n}, \quad e_{i}^{\prime} \in S .
$$

We define $\left(\tau\left(e_{0}\right), \ldots, \tau\left(e_{n}\right)\right)=\left(e_{0}^{\prime}, \ldots, e_{n}^{\prime}\right)$, which can be checked to be a generator sequence.

By iteration of $\tau$ on $\left(c_{0}, \ldots, c_{n}\right)$ we see that for large enough $m$ we have,

$$
\tau^{m}\left(c_{0}\right)=0, \quad \tau^{m}(V)=V+c \quad \text { for some } c \in S .
$$

The proof is done by taking $\left(c_{0}^{*}, \ldots, c_{n}^{*}\right)=\left(\tau^{m}\left(c_{0}\right), \ldots, \tau^{m}\left(c_{n}\right)\right)$ and $V+c=\tau^{m}(V)$.

Corollary 2. Let $T$ be an indeterminate over $C[Z]$. Let $\Phi: C[T][Z]$ $\rightarrow C[T][(f+T) / g]$ be the unique epimorphism which is identity on $C[T]$ and carries $Z$ to $(f+T) / g$. Clearly $\Phi$ has kernel $H-T$.

Let $C=k[u, v]$ as in Lemma 4. Then there exists $c \in k[u, T]$ such that $v+c=P(f+T)+Q g$ where $P=P(T), Q=Q(T) \in C[T]$.

Proof. Apply Lemma 5 with $S=k[u, T] / g, V=v$ and $h=f+T$; using Lemmas 3 and 4 to check the hypothesis.

COROllary 3. In the notation of Corollary 2 let $z^{*}=\Phi(Z), v^{*}=v+c$. Then we have

$$
\Phi(C[T][Z])=k\left[T, u, P z^{*}+Q\right] .
$$

Proof. Let $R=k\left[T, u, P z^{*}+Q\right]$. Since $g \in k[u] \subset R$ we get that $v^{*}$ $=g\left(P z^{*}+Q\right) \in R$. Also write $f+T=b_{0}^{*}+b_{1}^{*}\left(v^{*}\right)+\cdots+b_{n}^{*}\left(v^{*}\right)^{n}$ where $b_{i}^{*} \in k[T, u]$ and $b_{0}^{*} \in(g) k[T, u]$, as obtained by the application of Lemma 5 performed in Corollary 2 . Then

$$
z^{*}=\sum_{0}^{n} b_{i}^{*} g^{i-1}\left(P z^{*}+Q\right)^{i} \in R
$$

since $g, b_{0}^{*} g^{-1} \in k[T, u] \subset R$.

It is now clear that

$$
\Phi(C[T][Z])=\Phi(k[u, v, T, Z])=k\left[u, v, T, z^{*}\right]=k\left[u, v^{*}, T, z^{*}\right] \subset R \text {. }
$$

Since the opposite containment is clear, the proof is finished.

Proof of the TheORem. We claim that

$$
C[Z]=k[H, u, P(H) Z+Q(H)]
$$

and this will clearly establish the theorem. For proof, let $F \in C[Z]$. Then there exists a polynomial $F^{*}(X, Y, Z) \in k[X, Y, Z] \approx A(3, k)$ such that $\Phi(F)=F^{*}\left(T, u, P z^{*}+Q\right)$.

But then using $\Phi(T)=\Phi(H)$ we get that

$$
\Phi\left(F-F^{*}(H, u, P(H) Z+Q(H))\right)=0,
$$

i.e. 


$$
F-F^{*}(H, u, P(H) Z+Q(H))=(H-T) F^{* *},
$$

where $F^{* *} \in C[T][Z]$.

Putting $T=H$ on both sides we get $F=F^{*}(H, u, P(H) Z+Q(H))$. The claim now follows.

Remark. The above proof can be easily generalized to the following criterion for embedded hyperplanes. Let $T$ be an indeterminate over $A(n, k)$.

$H \in A(n, k)$ is an embedded hyperplane over $k \Leftrightarrow A(n, k)[T] /(H-T)$ $\approx A((n-1), k[T]) \Leftrightarrow A(n, k[T]) /(H-T) \approx A(n-1, k[T])$.

This also provides an alternative definition (4) in the introduction.

\section{REFERENCES}

[AEH] Shreeram S. Abhyankar, William Heinzer and Paul Eakin, On the uniqueness of the coefficient ring in a polynomial ring, J. Algebra 23 (1972). 310-342. MR 46 \# 5300.

[AM] Shreeram S. Abhyankar and Tzuong-tsieng Moh, Embeddings of the line in the plane, J. Reine Angew. Math. (to appear).

[R] Peter Russell, Simple birational extension of two dimensional affine rational domains (to appear).

Department of Mathematics, University of Kentucky, Lexington, Kentucky 40506 